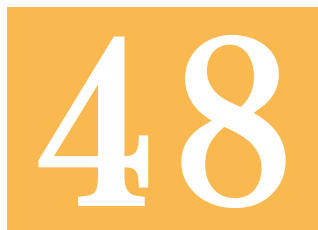

Año 18, N 48, San Salvador, El Salvador, Centroamérica. Revista Semestral Julio-Diciembre 2018

YEAR 18, N 48, SAN SALVADOR, EL SALVADOR, CENTRAL AMERICA. SEMESTRAL JOURNAL JuLY-DECEMBER 2018

\title{
Simulación de Retroiluminación Utilizando Diodo Emisor de Luz: Un Caso de Estudio en la Carretera CA-13
}

\section{Simulation of Retrofitting Utilizing Light Emitting Diode: A Case Study from CA-13 Roadway}

Omar Aragón Cuchilla

Investigador del Centro de Modelaje Matemático de la Universidad Francisco Gavidia PhD student, College of Engineering, National Central University (國立中央大學), Taiwan Master of Science, College of Engineering, National Central University (國立中央大學), Taiwan Ingeniería Industrial, Universidad Centroamericana José Simeón Cañas (UCA) maragon@ufg.edu.sv 104385602@cc.ncu.edu.tw

Paola Cruz Ceballos

Master of Science, College of Engineering. National Central University (國立中央大學) Taiwan Máster en Administración de Proyectos, Universidad Tecnológica Centroamericana (UNITEC), República de Honduras Ingeniería Civil, Universidad Católica de Honduras (UNICAH), República de Honduras cruzpaola2010@gmail.com

Bryon Sheldon Flowers $\mathrm{PhD}$ student, Department of Bioenvironmental Systems Engineering, National Taiwan University (國立中央大學), Taiwan Master of Science, College of Engineering, National Central University (國立中央大學), Taiwan Bachelor of Science, Faculty of Science and Technology, University of Belize bryonflowers@outlook.com

\section{RESUMEN}

Este artículo hace una simulación de retroiluminación utilizando diodo emisor de luz (LED) en sustitución de iluminación con lámparas de sodio de alta presión (HPS), además presenta un análisis costo-beneficio evaluando diversos aspectos técnicos y económicos del caso de estudio. La simulación de la retroiluminación fue ejecutada con el software DIAlux. Para llevar a cabo el estudio, se seleccionó la carretera CA-13 en La Ceiba, Honduras, la cual es una autopista de interconexión con el océano Atlántico centroamericano. El estudio tuvo como objetivo estimar si se satisfacen los requisitos técnicos y económicos para la retroadaptación de las estructuras de iluminación en el área de estudio. 
Los hallazgos del estudio demuestran que, aunque los costos iniciales de la retroadaptación de la iluminación tradicional, utilizando diodo emisor de luz son altos, los ahorros de energía y reducción en gastos por mantenimiento son significativos, siendo estas las principales motivaciones de por qué muchas ciudades conducen proyectos de carreteras con tecnología LED. Para el caso propuesto, la energía ahorrada es de $233 \mathrm{MWh}$ (53.3\%), en términos de dinero los ahorros ascienden a USD 38,987 anualmente en comparación con las lámparas de sodio de alta presión. Financieramente, este es un proyecto con un periodo de amortización de 5 años, el VPN posee un valor positivo de USD 196,689, y hay una TIR alta de $20.5 \%$, siendo un proyecto atractivo para potenciales inversores. Además, la relación costo-beneficio es de 1.83; por lo tanto, el proyecto se considera viable. Adicionalmente, la reducción de las emisiones de dióxido de carbono con la implementación de una tecnología de energía limpia se estima en 77.5 toneladas de $\mathrm{CO}^{2}$ anualmente.

En conclusión, las luces LED para iluminación de carreteras, ofrecen una solución eficaz y cada vez más rentable al desafío del desarrollo del consumo de energía municipal, de los gastos de la red de luz carreteras, de la reducción de emisiones de gases de efecto invernadero y de la calidad urbana.

Palabras clave: retroiluminación, diodo emisor de luz, ahorro energético, emisiones de dióxido de carbono, carreteras.

\section{ABSTRACT}

This article performs a retrofitting simulation approach using light emitting diode instead of sodium high pressure luminaries; in addition, executes a cost-benefit analysis evaluating technical and economical aspects from the case of study. The illuminance simulation was executed by DIAlux. For performing the study, CA-13 roadway, La Ceiba, Honduras, was selected. The studied roadway is extremely relevant considering that connects with part of the Central American Atlantic region.

Findings from the study demonstrate that although the initial costs of retrofitting conventional lighting to LED are high, the energy and maintenance savings are considerable, and these are the main motivations for why many cities worldwide conduct LED street lighting projects. For the proposed case, the energy consumption saved accounts for $233 \mathrm{MWh}(53.3 \%)$ annually. Monetarily, savings ascend to USD 38,987 annually compared to the usage of high-pressure sodium lamps (HPS). Financially, this is a viable project, the payback is in 5 years, the NPV is a positive value of USD 196,689, and there is a high IRR of 20.5\%, being an attractive percentage for potential investors. Moreover, the benefit-cost ratio equals to 1.83, this value being greater than one, indicates that the project is economically viable. Finally, results from this research indicate that the reduction of GHG emissions by implementing the LED lighting technology would be $77.5 \mathrm{t} \mathrm{CO}_{2}$ annually.

In conclusion, LED roadway lights offer an effective and increasingly cost-effective solution to the development challenge of municipal energy consumption, roadway light network expenditures, greenhouse gases emissions reduction and urban quality.

Keywords: illuminance retrofitting, light emitting diode, energy saving, carbon dioxide emissions, roads.

\section{Introducción}

La iluminación vial es un servicio público clave proporcionado por las autoridades a nivel local y municipal. Estudios previos han demostrado que el nivel de iluminación vial es un factor importante para la seguridad vial, la seguridad personal y el ambiente urbano (Zhou et al., 2009). La iluminación vial asegura la visibilidad en la oscuridad para los conductores, ciclistas y peatones, reduciendo así la probabilidad de accidentes. A la vez, facilita indirectamente la prevención del delito aumentando el sentido de seguridad personal, así como la seguridad de las propiedades públicas y privadas adyacentes (Dewar and Olson, 2007). Los efectos de iluminación vial también pueden impulsar el atractivo de las ciudades, pueblos y comunidades, destacar atractivos locales o acentuar la atmósfera durante importantes eventos públicos (Solentis Engineering Light, 2018).

Sin embargo, muchas instalaciones de alumbrado vial son obsoletas y por lo tanto, 
altamente ineficientes. Esto conduce a mayores requerimientos de energía y niveles de mantenimiento (EU Smart Cities Information System, 2018). El Gobierno de Honduras anunció recientemente el aumento de la tarifa eléctrica, por lo que el costo operacional de la luz vial será mucho mayor (La Prensa de Honduras, 2018). La luz tradicional de carreteras tiene un período de duración de vida corto, entre 12,000 horas hasta 24,000 horas (Colon, 2010).

Por otro lado, al llevar a cabo un análisis de factibilidad deben tomarse en cuenta otros factores como la ineficiencia de los sistemas de iluminación vial tradicional con lámparas de sodio de alta presión (HPS, siglas en inglés), los cuales contribuyen a una mayor emisión de gases de efecto invernadero debido a que consumen una alta cantidad de energía para producir la misma iluminancia comparada a los sistemas de iluminación que usan diodo emisor de luz (LED, siglas en inglés) (Colon, 2010).

El propósito de este artículo es investigar si la retroiluminación vial con tecnología LED puede ayudar a ahorrar energía, reducir los costos y mejorar la calidad ambiental. También, proporcionar un estudio de factibilidad para mostrar cómo la tecnología LED puede ser económicamente rentable y puede aportar a mejorar la calidad de vida de las poblaciones.

\section{Método}

Con el objetivo de justificar la retroiluminación utilizando diodo emisor de luz en la carretera CA-13 de La Ceiba, Honduras; se especificaron los parámetros necesarios a estimar para la iluminación vial, se realizó una simulación del estado actual de la calzada de la carretera y del estado posterior a la retroiluminación utilizando el software de creación alemana DIAlux y finalmente se llevó a cabo un análisis costo-beneficio para estimar la rentabilidad financiera del estudio. Los requisitos, parámetros y especificaciones utilizados para los análisis de retroiluminación y rentabilidad en esta investigación; fueron obtenidos de la guía de luminotecnia de la Universidad Mayor de San Simón (UMSS), Cochabamba, Bolivia (Maldonado, 2001). Esta guía está siendo utilizada actualmente por la Empresa Energía de Honduras (EEH) para el diseño y consideraciones técnicas del alumbrado en la red de Honduras. Los procesos que se llevaron a cabo en esta investigación se detallan en el diagrama de flujo representado en la Figura n. ${ }^{\circ} 1$.

\section{Iluminación vial}

El objetivo primordial de la iluminación vial es proporcionar alumbrado de calidad para brindar seguridad y confort a los conductores y transeúntes. Los parámetros y valores necesarios para estimar la calidad de la iluminación vial en este estudio fueron especificados con base en el estudio realizado por Maldonado, 2001. Los parámetros se especifican en la Tabla 1 y en la Tabla 2; adicionalmente, a continuación, se describe cada uno de ellos de forma más específica:

\section{Iluminancia}

Se define iluminancia como el flujo luminoso recibido por una superficie medida en lux, el cual indica la iluminación en un punto sobre una superficie que dista, en dirección perpendicular, un metro de una fuente puntual uniforme de 


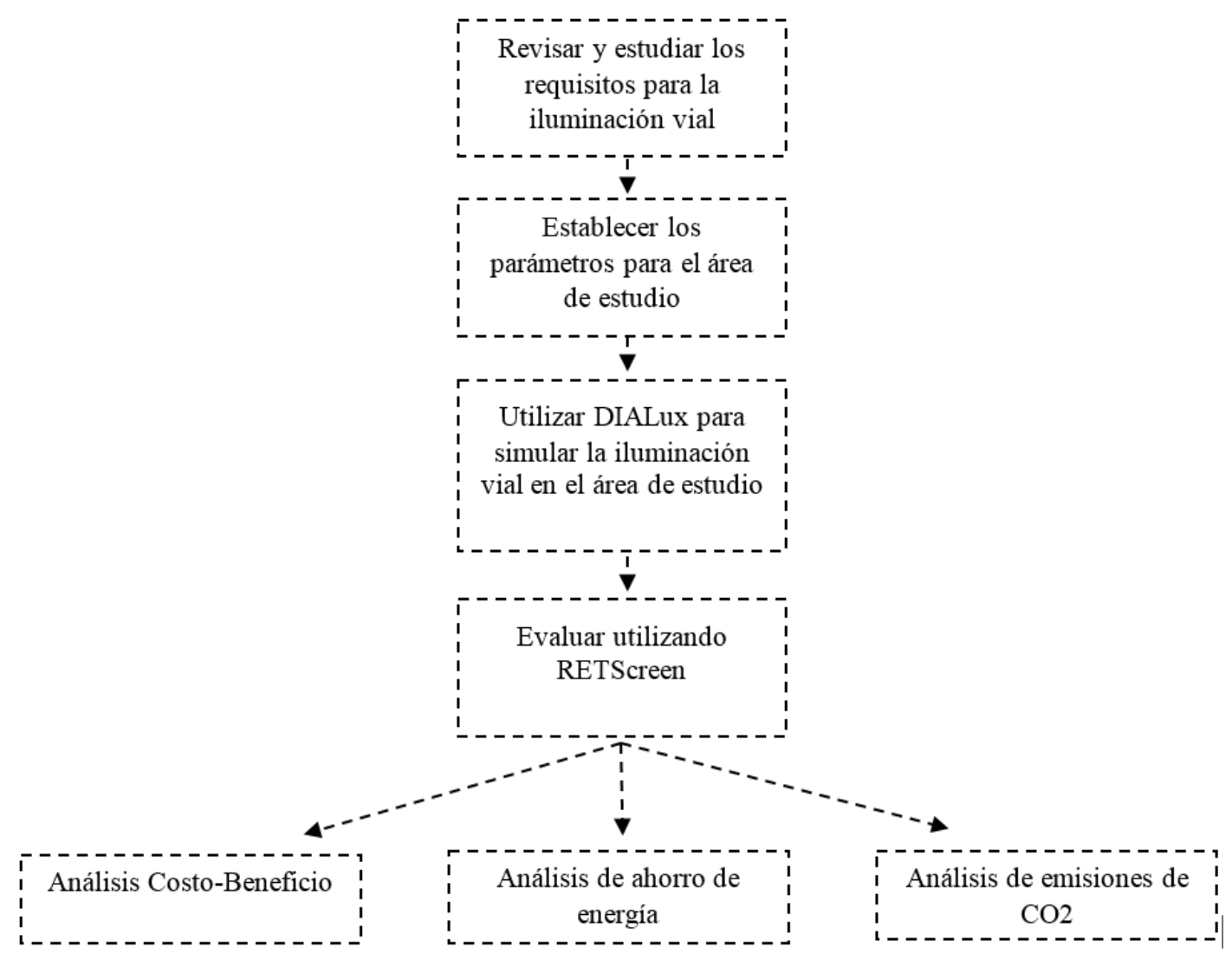

Figura $n .^{\circ}$ 1. Diagrama de flujo de los procesos de investigación. Fuente: elaboración propia.

una candela (Comisión Internacional de la Iluminación, 2011; Maldonado, 2001).

$$
E=\frac{d \emptyset}{d S}
$$

donde, $d \phi$ representa el flujo luminoso y $d s$ la superficie sobre la que actúa el flujo luminoso.

\section{$\underline{\text { Luminancia }}$}

Se determina luminancia a la relación entre la intensidad luminosa y la superficie aparente vista por el ojo humano en una dirección determinada (Maldonado, 2001).

$$
L=\frac{I}{S_{\text {aparente }}}
$$

(2)

donde, I determina la intensidad luminosa medida en candelas o lúmenes; y $S_{\text {aparente }}$ determina el área sobre la cual está actuando la fuente de luz. Por otro lado, la luminancia media $\left(L_{a v}\right)$ es un parámetro que depende de las características del tipo de pavimento, 
clase de vía, intensidad del tráfico, entre otros (Maldonado, 2001).

\section{$\underline{\text { Coeficientes de uniformidad }}$}

El rendimiento visual es un parámetro que se estima mediante el coeficiente global de uniformidad $U_{0}$ (2); adicionalmente, la comodidad visual se estima mediante el coeficiente longitudinal de uniformidad $U_{I}$ (3) (Maldonado, 2001).

$$
U_{0}=\frac{L_{\min }}{L_{m}}
$$

$$
U_{I}=\frac{L_{\min }}{L_{\max }}
$$

En general, la uniformidad de la iluminación es inversamente proporcional al espaciamiento de las luminarias, el cual repercute en los costos totales de la iluminación vial.

\section{$\underline{\text { Incremento del umbral }}$}

Incremento del umbral se entiende como la condición de la visión durante la cual se sufre un daño o una reducción de la capacidad de distinguir los detalles de un objeto debido a una distribución desfavorable de las luminarias o por contrastes excesivos (Comisión Internacional de la Iluminación, 2011). El incremento del umbral es un parámetro adimensional medido como una proporción. Con base en los efectos que produce el incremento del umbral, puede clasificarse en incremento del umbral insoportable, molesto, admisible, satisfactorio e inapreciable (Maldonado, 2001).

Tabla n. ${ }^{\circ} 1$

Recomendaciones Comisión Internacional de la Iluminación para el alumbrado público

\begin{tabular}{|c|c|c|c|}
\hline \multirow{2}{*}{ Clase de vía } & \multirow{2}{*}{ Tipo de alrededores } & \multicolumn{2}{|c|}{ Deslumbramiento } \\
\cline { 2 - 4 } & & Molesto (G) & Perturbador (TI) \\
\hline Autopista & Cualquiera & 6 & 5 \\
\hline \multirow{2}{*}{ Carretera -arteria- } & Claro & 6 & 5 \\
\cline { 2 - 4 } & Oscuro & 5 & 20 \\
\hline \multirow{2}{*}{ Carretera -principal- } & Claro & 6 & 5 \\
\cline { 2 - 4 } & Oscuro & 4 & 20 \\
\hline Calle comercial & Claro & 4 & 20 \\
\hline \multirow{2}{*}{ Calle de interconexión } & Claro & 5 & 10 \\
\cline { 2 - 4 } & Oscuro & 5 & 20 \\
\hline
\end{tabular}

Fuente:(Maldonado, 2001). 


\section{$\underline{\text { Relación circundante }}$}

La relación circundante ( $S R$ ) es una medida de la iluminación en las zonas limítrofes de una vía (5 metros), tiene por objetivo cuantificar la visibilidad de los objetos o seres humanos en la zona limítrofe de una vía (Maldonado, 2001).

\section{$\underline{\text { Iluminancia media }}$}

La iluminancia media ( $E_{A V}$ ) especifica el nivel de iluminancia promedio de un área específica, la cual depende de las características de la superficie, clase de vía, intensidad del tráfico, entre otros (Maldonado, 2001).

$$
E_{A V}=\frac{\rho \varphi_{i} n}{w d}
$$

donde, $\rho$ representa el factor de utilización de la instalación; $\varphi_{i}$ es el flujo luminoso de la lampara; $n$ es el número de lámparas por luminaria; $w$ es la anchura por iluminar de la calzada y $\mathrm{d}$ representa la separación entre luminarias.

\section{Simulación utilizando DIAlux}

DIAlux es un software libre que se utiliza para diseñar, calcular y visualizar la iluminación en habitaciones individuales, pisos enteros, edificios y escenas al aire libre. DIAlux es utilizado como herramienta de planeamiento que satisface los requisitos del diseño moderno de la iluminación y del cálculo de la iluminación. Además, utiliza catálogos de luminarias electrónicas de los fabricantes de luminarias líderes del mundo.
Para estimar de forma más precisa el tipo de luz LED acorde a las condiciones de la carretera CA-13 en La Ceiba, Honduras; fue necesario inicialmente, simular la actual calzada de la carretera con las lámparas HPS, para obtener valores existentes de luminancia y luminosidad con DIAlux. A continuación, se describen los pasos que se siguieron para obtener un sistema de iluminación óptimo a partir de la posición actual de los postes y de las fuentes de luz existentes.

1. Determinar los valores de los parámetros de iluminación y compararlos con los valores recomendados por las normas vigentes (ver Tablas n. 2 a la 4 ).

Tabla n. ${ }^{\circ} 2$

Características de carretera para diseñar en DIAlux

\begin{tabular}{|l|c|}
\hline \multicolumn{1}{|c|}{ Descripción } & Unidad de Medida \\
\hline Ancho de carretera & Pie \\
\hline Distancia del poste & Pie \\
\hline Altura de montaje & Pie \\
\hline Longitud del brazo & Pie \\
\hline Angulo de incidencia & Grados \\
\hline Posicionamiento de los postes & $\begin{array}{c}\text { Centro, una cara, doble } \\
\text { cara, doble cara con } \\
\text { desplazamiento }\end{array}$ \\
\hline
\end{tabular}

Fuente:(Maldonado, 2001). 
Tabla n. 3

Factores de luminancia

\begin{tabular}{|c|l|c|}
\hline \multicolumn{2}{|c|}{ Factores de luminancia } & Valor del parámetro \\
\hline $\mathrm{L}_{\mathrm{av}}$ & $\begin{array}{l}\text { Luminancia media de la superficie de la carretera (de una calzada de } \\
\text { una carretera) } \mathrm{cd} / \mathrm{m}^{2}\end{array}$ & $\geq 0.80$ \\
\hline $\mathrm{U}_{0}$ & Uniformidad total (de la luminancia de la superficie de la carretera) & $\geq 0.40$ \\
\hline $\mathrm{U}_{\mathrm{I}}$ & $\begin{array}{l}\text { Uniformidad longitudinal (de la luminancia de la superficie de una } \\
\text { calzada de la carretera) }\end{array}$ & $\leq 15$ \\
\hline $\mathrm{T}_{\mathrm{I}}$ & Incremento del umbral (\%) & $\geq 0.5$ \\
\hline $\mathrm{SR}$ & Relación circundante (de la iluminación de una calzada de un camino) & \\
\hline
\end{tabular}

Fuente:(Maldonado, 2001).

Tabla n. ${ }^{\circ} 4$

Factores de iluminación

\begin{tabular}{|c|l|c|}
\hline \multicolumn{1}{|c|}{ Factores de luminancia } & Valor del parámetro \\
\hline $\mathrm{EAV}$ & $\begin{array}{l}\text { Iluminación media de la superficie de la carretera (de una } \\
\text { calzada de carretera) lx }\end{array}$ & 15 \\
\hline $\mathrm{u}_{0}$ & Uniformidad de la iluminación de la superficie vial & 0.4 \\
\hline $\mathrm{E}_{\min } / \mathrm{E}_{\max }$ & Iluminación del cociente de la iluminación superficial de la carretera & 0.33 \\
\hline
\end{tabular}

Fuente:(Maldonado, 2001).

2. Obtener las dimensiones de la calzada y las características del sistema de iluminación medidos en el campo (ver Figura n. ${ }^{\circ} 2$ ).

3. Recrear el proyecto de diseño original utilizando luminaria actual y las características reales de campo (dimensiones de calle, postes y posición de postes).Tal como se observa en las Figuras n. 3 y 4, los valores de los parámetros de iluminación en este diseño son en realidad muy pequeños en comparación con los que figuran en las normas y estándares de iluminación actuales. El requisito de luminancia se cumple, pero no los coeficientes de uniformidad que están por debajo del valor estándar. Es notable la mala iluminación y la baja calidad de la luminancia. Muestra de ello son las isolíneas de iluminación, E, obtenidas con un diseño que utiliza las características de la calle y del sistema de iluminación. La iluminancia promedio (Eav = 21) está por debajo del valor estándar y las isolíneas no están distribuidas uniformemente a lo largo de la carretera.

La Figura n. 5 muestra la iluminación simulada de un tramo de carretera con luminaria GELIGHTING 400W HPS en posición de desplazamiento. En dicha figura, se puede observar la pequeña cantidad de luz reflejada en el pavimento, además se observa que hay muchas áreas oscuras en todo el camino que muestran desproporción en la iluminación. De igual forma, se puede apreciar el render de color falso siendo esta el área alrededor del poste donde la iluminación es "más brillante" 


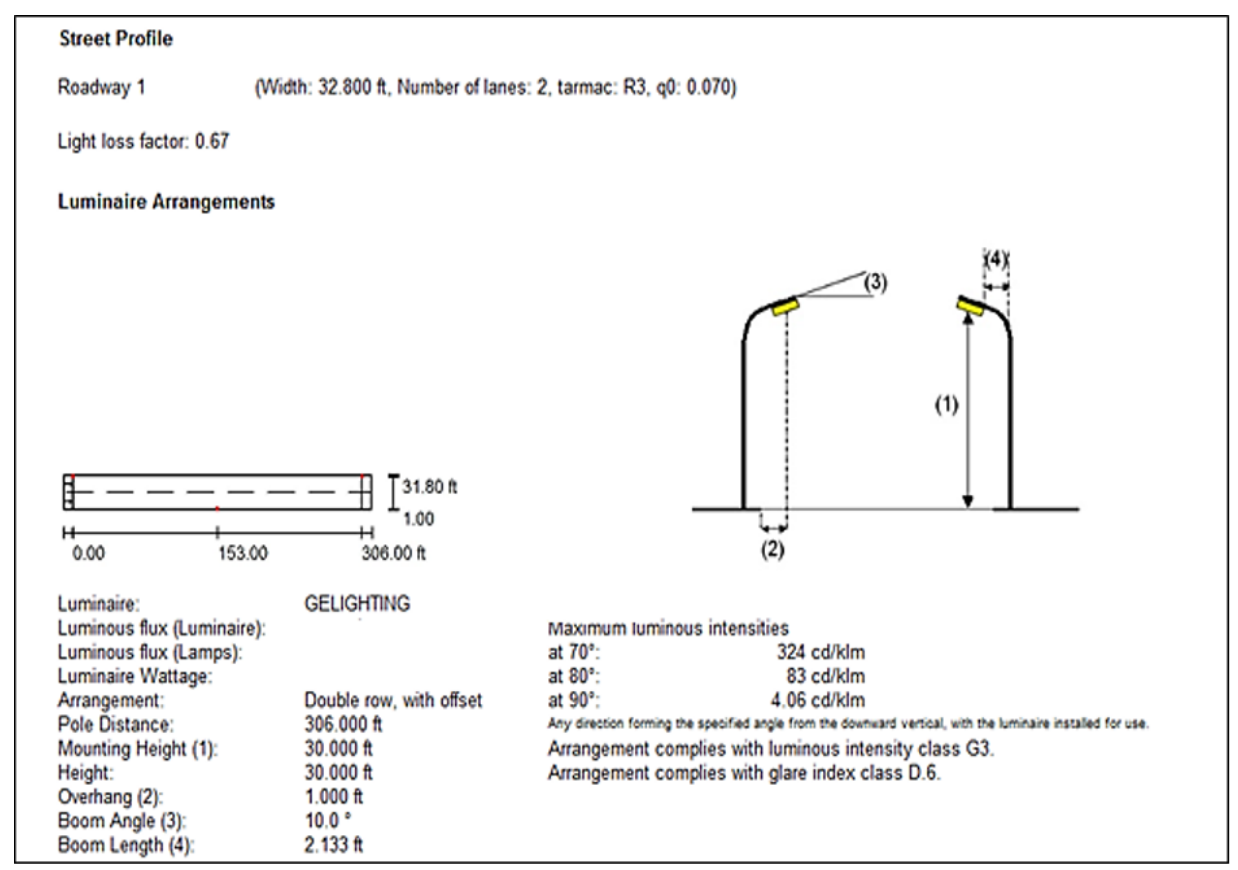

Figura $n .^{\circ}$ 2. Perfil y características de luminaria en la carretera CA-13. Fuente: DIAlux

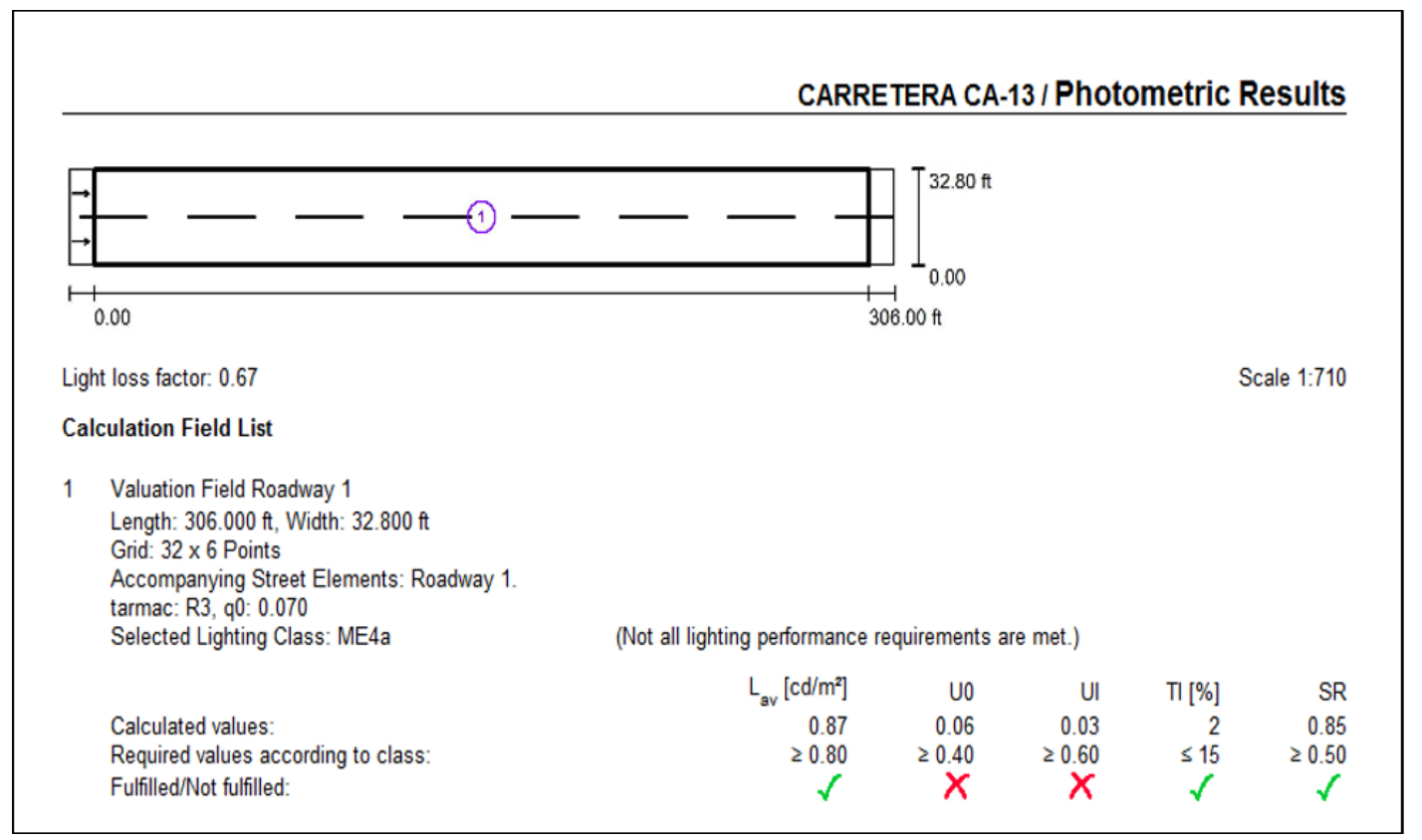

Figura $n .{ }^{\circ}$ 3. Resultados fotométricos con luminaria HPS de 400W. Fuente: DIAlux. 
CARRETERA CA-13 / Valuation Field Roadway 1 / Isolines (E)

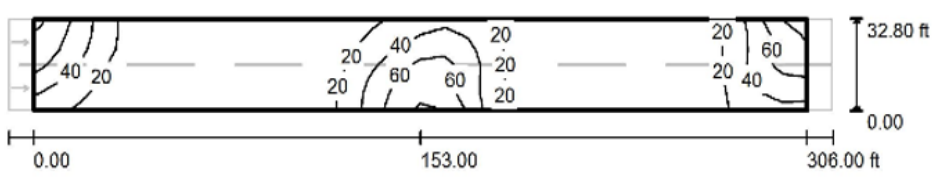

Values in Lux, Scale $1: 710$

Grid: $32 \times 6$ Points

$\begin{array}{rrrrr}\mathrm{E}_{\mathrm{av}}[\mathrm{lx}] & \mathrm{E}_{\min }[\mathrm{lx}] & \mathrm{u} & \mathrm{E}_{\min } / \mathrm{E}_{\max }[\mathrm{lx}] \\ 21 & 0.74 & 83 & 0.035 & 0.009\end{array}$

Figura $n .^{\circ}$ 4. Isolíneas con luminaria HPS de 400W. Fuente: DIAlux.

o donde la luz tiene más intensidad, observándose una desproporción en la iluminación.

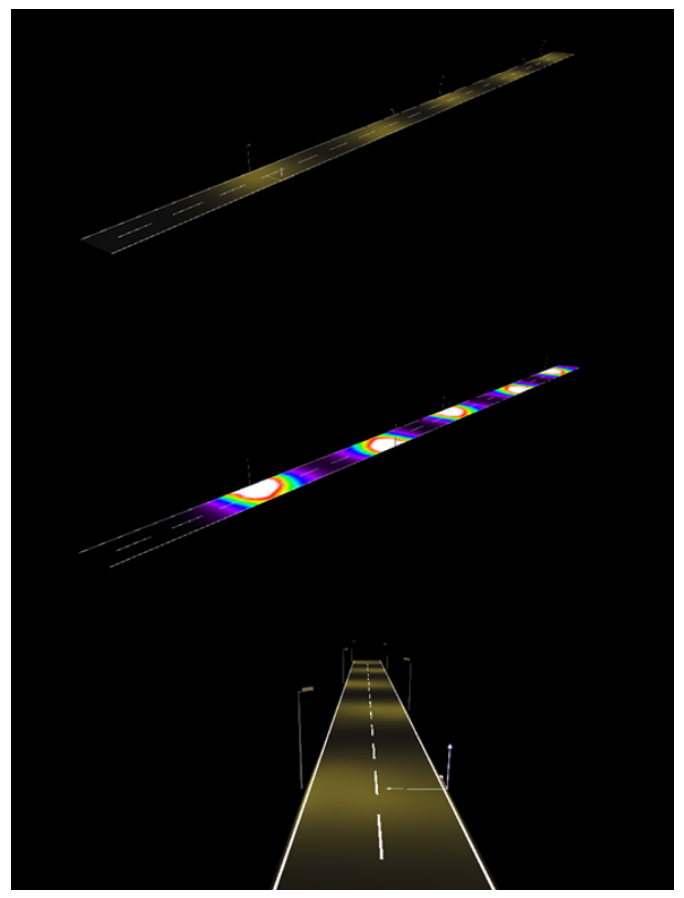

Figura $n{ }^{\circ}$ 5. Simulación de la carretera CA13 con luminaria existente de 400W. Fuente: DIAlux.
4. Recrear nuevamente la calzada manteniendo las características de campo (tipo de calle, postes y posición de los postes) utilizando los diferentes sistemas de iluminación LED con la ayuda del catálogo electrónico proporcionado por DIAlux.

\section{Análisis RETScreen}

En esta investigación se realizó un análisis con el software RETScreen con el fin de estimar los valores óptimos de las variables necesarias a estimar en función de la factibilidad de costos, ahorro de energía y reducción de emisiones de dióxido de carbono, para la retroiluminación de las estructuras de la carretera CA-13 en La Ceiba, Honduras, con iluminación tipo LED. RETScreen es un sistema de software de gestión de energía limpia para el análisis de viabilidad de proyectos de eficiencia energética, energía renovable y cogeneración, así como de análisis de rendimiento energético en curso (RETScreen, 2018).

Para realizar el análisis, inicialmente se infirió información general sobre el proyecto (tipo de 
proyecto, tipo de instalación, tipo de análisis), luego, se determinan los factores climáticos de la región de estudio. RETScreen consiste en una serie de hojas de cálculo y bases de datos que permiten al usuario crear, importar datos, estimar, calcular y filtrar conjuntos de datos a utilizar en el análisis. A continuación, se detalla cada uno de ellos:

- La hoja de cálculo del modelo de energía se utiliza para simular el consumoy/o la producción de energía de diversos tipos de instalaciones, incluyendo medidas y sistemas individuales.

- La hoja de cálculo de costos se utiliza para estimar los costos de un caso propuesto. Los costos se presentan desde perspectivas iniciales y recurrentes.

- La hoja de cálculo de análisis de reducción de emisiones se utiliza para estimar las emisiones de gases de efecto invernadero de un proyecto propuesto. Tiene cuatro secciones principales:

1. Configuración: el usuario especifica el tipo de análisis realizado y proporciona factores potenciales de calentamiento global;

2. Caso base del sistema eléctrico y el caso base del resumen del sistema de gases invernaderos: se proporciona una descripción de la línea base de los gases de efecto invernadero para un sistema existente;

3. Resumen del sistema de gases de efecto invernadero propuesto del caso propuesto (proyecto): proporciona al usuario un perfil de emisiones del proyecto propuesto;
4. Un resumen de reducción de emisiones de gas de efecto invernadero: los resultados de la hoja de cálculo se presentan en toneladas equivalentes de dióxido de carbono evitados por año.

- La hoja de cálculo de análisis financiero contiene seis secciones que proporcionan información sobre el flujo de ingresos del proyecto. Las secciones incluidas son: parámetros financieros, ingresos anuales, costos del proyecto y resumen de ahorros/ ingresos, viabilidad financiera, flujos de efectivo anuales y gráficos de flujos de efectivo acumulados.

- Análisis de sensibilidad y riesgo (opcional): identifica cómo la incertidumbre en las estimaciones de varios parámetros clave puede afectar la viabilidad financiera del proyecto. Utiliza la simulación de Monte Carlo, técnica matemática computarizada que permite a las personas dar cuenta del riesgo en el análisis cuantitativo y la toma de decisiones. Proporciona a la toma de decisiones una serie de posibles resultados y las probabilidades de que se produzcan para cualquier elección de acción (Palisade, 1984). Además, el análisis de sensibilidad proporciona un gráfico de tornado que muestra un análisis de sensibilidad comparativa. Está pensado para darle al analista una idea de que factores son más importantes al problema de decisión/riesgo en cuestión. También es útil como parte de los resultados analíticos del proyecto, dando a los tomadores de decisiones una idea de las incertidumbres y su potencial impacto (Dalton, 2012). 
Los siguientes datos de entrada se analizaron mediante las diferentes hojas de cálculo de RETScreen en esta investigación:

- Horas diarias de funcionamiento.

- Tarifa eléctrica (USD \$/kWh).

- Inventario existente de la estructura de la iluminación (por tecnología y por potencia).

- Reemplazo de la tecnología de luz vial, incluyendo potencia (vatios) de reemplazo.

- Costo de instalación (USD).

- Costo de mantenimiento por tipo de luminaria (USD/Unidad/año).

- Tasa de inflación (\%).

- Tasa de descuento (\%).

\section{Resultados y Discusión}

En esta sección se muestran y discuten los resultados obtenidos con la simulación utilizando los softwares DIAlux y RETScreen.

\section{DIAlux}

En la presente sección se proporciona una tabla resumen con los valores de los distintos parámetros obtenidos con la simulación realizada utilizando DIAlux (ver Tabla n. ${ }^{\circ}$ 5). Los números en rojo remarcan aquellos parámetros cuyo valor no se encuentra dentro de los valores aceptables en las especificaciones. Cabe destacar que los valores de luminancia e iluminación en los tres tipos de luminarias HPS se encuentran por debajo de las especificaciones establecidas.

Según se observa en la Tabla $n .^{\circ}$ 5, las tres luminarias LED (70W, $150 \mathrm{~W}$ y $200 \mathrm{~W}$ ) estimadas en la simulación, brindan valores de luminancia e iluminación dentro de los rangos establecidos en las especificaciones; sin embargo, cabe destacar que los factores de luminancia y de iluminación no son los únicos parámetros a tener en cuenta en la toma de decisión para determinar la opción óptima en el área de estudio. Adicionalmente, deben de estimarse parámetros que midan la calidad y los costos de los sistemas de luminarias.

Por ejemplo, la luminaria LED de 200W, brinda los valores de luminancia e iluminación más altos en comparación con los otros sistemas de luminarias; sin embargo, el precio de esta luminaria es de aproximadamente USD 1,000 por lámpara (Wu et al., 2009). En el caso de la luminaria LED de $150 \mathrm{~W}$ brinda valores de luminancia e iluminación inferiores a los obtenidos con la luminaria LED de 200W; no obstante, su precio es de aproximadamente USD 760 por lámpara (Tähkämö et al., 2016). Por otro lado, el sistema de luminaria LED de $70 \mathrm{~W}$ tiene un precio aproximado de USD 300 por lámpara (Philips, 2017), y brinda valores de uniformidad de luminancia superiores a los sistemas de $150 \mathrm{~W}$ y $200 \mathrm{~W}$. Sin embargo, brinda valores de iluminación media de la superficie de la carretera y de uniformidad de la iluminación de la superficie vial inferiores a los valores obtenidos en los otros dos sistemas. Para el caso del presente estudio, los parámetros con más relevancia en la investigación son los parámetros que miden la iluminación, debido a que se desea 
Tabla n. ${ }^{\circ} 5$

Resumen de simulación en DIAlux para luminarias HPS y LED

\begin{tabular}{|c|c|c|c|c|c|}
\hline & & \multicolumn{4}{|c|}{ Tipo de luminaria } \\
\hline \multicolumn{2}{|c|}{ Parámetros } & 400W HPS & 200W LED & 150W LED & 70W LED \\
\hline \multirow{5}{*}{ Luminancia } & $\mathrm{Lav}(\mathrm{cd} / \mathrm{m} 2)(\geq 0.80)$ & 0.87 & 2.16 & 1.25 & 0.99 \\
\hline & U0 $(\geq 0.40)$ & 0.06 & 0.63 & 0.61 & 0.66 \\
\hline & UI $(\geq 0.60)$ & 0.03 & 0.65 & 0.65 & 0.80 \\
\hline & $\mathrm{TI}(\%)(\leq 15)$ & 2 & 12 & 10 & 8 \\
\hline & $\mathrm{SR}(\geq 0.5)$ & 0.85 & 0.50 & 0.58 & 0.52 \\
\hline \multirow[t]{5}{*}{ Illuminación } & $\mathrm{E}_{\mathrm{av}}(\mathrm{lx})(\geq 15)$ & 21 & 32 & 18 & 15 \\
\hline & $\mathrm{E}_{\min }(\mathrm{lx})$ & 0.74 & 20 & 10 & 8.06 \\
\hline & $\mathrm{E}_{\max }(\mathrm{lx})$ & 83 & 46 & 32 & 22 \\
\hline & U0 ( $\geq 0.4)$ & 0.035 & 0.635 & 0.562 & 0.555 \\
\hline & $\mathrm{Emin} / \max (\geq 0.33)$ & 0.009 & 0.443 & 0.319 & 0.368 \\
\hline Vida útil & Horas & 12,000 & 50,000 & 50,000 & 50,000 \\
\hline Marca & & GE & Philips & Philips & Philips \\
\hline Salida de luz & Cociente & 0.76 & 0.81 & 0.76 & 0.71 \\
\hline Dimensiones & Metros $(\mathrm{LxBxH})$ & $\begin{array}{c}0.83 \mathrm{~m} \mathrm{x} \\
0.35 \mathrm{~m} \mathrm{x} \\
0.22 \mathrm{~m}\end{array}$ & $\begin{array}{c}0.71 \mathrm{~m} \mathrm{x} \\
0.36 \mathrm{~m} \mathrm{x} \\
0.10 \mathrm{~m}\end{array}$ & $\begin{array}{c}0.71 \mathrm{~m} \mathrm{x} \\
0.36 \mathrm{~m} \mathrm{x} \\
0.10 \mathrm{~m}\end{array}$ & $\begin{array}{c}0.71 \mathrm{~m} \mathrm{x} \\
0.36 \mathrm{~m} x \\
0.10 \mathrm{~m}\end{array}$ \\
\hline
\end{tabular}

Fuente: elaboración propia, datos tomados de los resultados generados por DIAlux.

determinar el sistema de luminaria que brinde mayor y más uniforme iluminación a los usuarios con un razonable costo.

La Figura n. 6 muestra los resultados fotométricos de la iluminación del camino con la luminaria LED de $150 \mathrm{~W}$. Se puede observar que los valores de los parámetros de iluminación en este diseño cumplen con todas las normas y estándares de iluminación. Por otro lado, el valor de luminancia con la luminaria LED de $150 \mathrm{~W}$ resulta más alto que el valor obtenido con la luminaria HPS de $400 \mathrm{~W}$, lo que hace que la carretera sea más visible; adicionalmente, los coeficientes de uniformidad con la luminaria LED de $150 \mathrm{~W}$ en comparación con la luminaria HPS de 400W resultan ser más altos y, por lo tanto, en la simulación la carretera resulta estar completamente iluminada. 


\section{CARRETERA CA-13/Photometric Results}

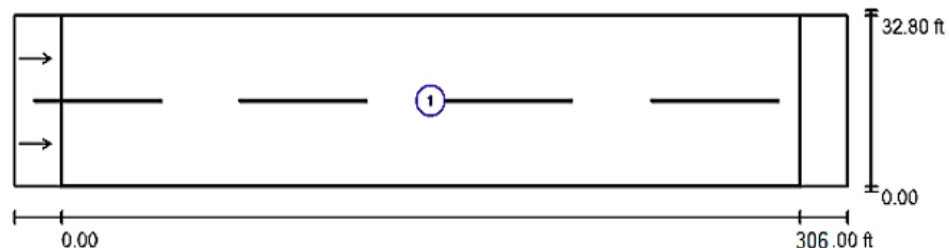

Light loss factor: 0.67

\section{Calculation Field List}

1 CARRETERA CA-13

Length: $306.000 \mathrm{ft}$, Width: $32.800 \mathrm{ft}$

Grid: $16 \times 6$ Points

Accompanying Street Elements: CARRETERA CA-13.

tarmac: R3, q0: 0.070

Selected Lighting Class: ME4a

Calculated values:

Required values according to class:

Fulfilled/Not fulfilled:

(All lighting performance requirements are met.)

$\begin{array}{rrrrr}\mathrm{L}_{\mathrm{av}}\left[\mathrm{cd} / \mathrm{m}^{2}\right] & \mathrm{U} & \mathrm{Ul} & \mathrm{Tl}[\%] & \mathrm{SR} \\ 1.25 & 0.61 & 0.65 & 10 & 0.58 \\ 20.80 & 20.40 & 20.60 & \leq 15 & 20.50 \\ \checkmark & \checkmark & \checkmark & \checkmark & \checkmark\end{array}$

Figura $n .{ }^{\circ}$ 6. Resultados fotométricos con luminaria LED de 150W. Fuente: DIAlux.

En la Figura n. ${ }^{\circ}$, también se observa una alta calidad deiluminaciónyluminancia,que muestran las isolíneas de iluminación, E, obtenidas con un diseño que utiliza las características de la calle y del sistema de iluminación usando la luminaria
LED de 150W. La iluminancia promedio (Eav = 18) cumple con el valor estándar y las isolíneas se distribuyen de manera más uniforme en toda la calzada en comparación con el sistema HPS de 400W.

\section{CARRETERA CA-13 / CARRETERA CA-13/Isolines (E)}

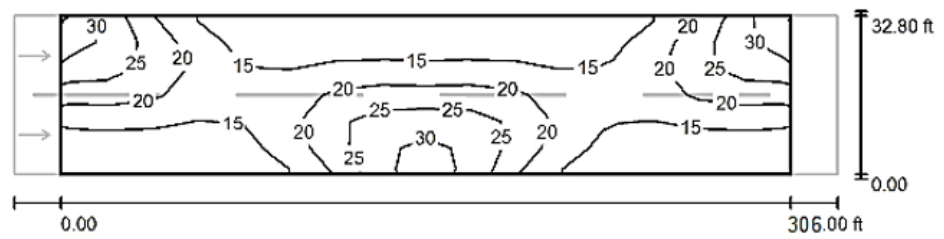

Values in Lux, Scale 1: 377

Grid: $16 \times 6$ Points
$E_{a v}[l x]$
$E_{\min }[\mid x]$
$E_{\max }[\mathrm{lx}]$
u0
0.562
$\mathrm{E}_{\min } / \mathrm{E}_{\max }$ 0.319

Figura $n .^{\circ} 7$. Isolíneas con luminaria LED de $150 \mathrm{~W}$. 
La Figura n. ${ }^{\circ}$ 8, muestra un tramo simulado de la carretera CA-13 en La Ceiba, Honduras, iluminado con una luminaria Philips 150W LED en posición de desplazamiento. Se puede observar uniformidad continua en la iluminación del camino. Además, se observa claramente el render de color falso en donde la intensidad de la luz es realmente alta en comparación con las luminarias HPS de 400W y de la luminaria LED de 70W.

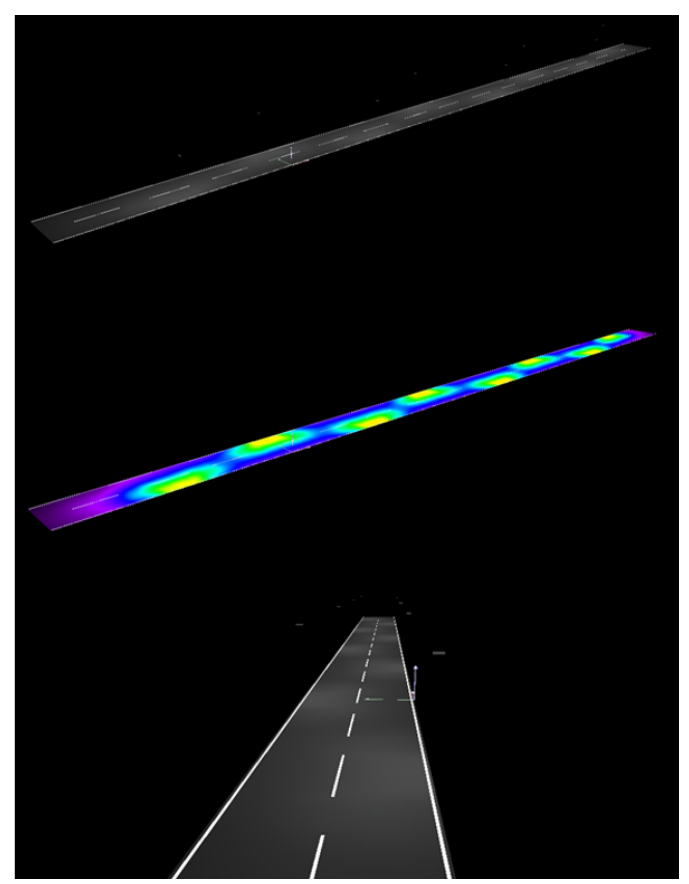

Figura $n .^{\circ}$ 8.Simulación de la carretera CA-13 con luminaria LED de 150W. Fuente: DIAlux.

Teniendo en cuentalas anteriores consideraciones, se determinó que la luminaria LED de $150 \mathrm{~W}$ cumple con la calidad de la iluminación y precio de sustitución de todas las lámparas de 400W HPS en el área de estudio de la carretera CA-13 en La Ceiba, Honduras.

\section{RETScreen}

Los datos de entrada que se usaron para realizar el análisis con el software RETScreen fueron obtenidos de diversas fuentes. La tasa de inflación y la tasa de descuento fueron obtenidos del Banco Central de Honduras; la tarifa eléctrica, horas y días de operación, la tarifa escalada del costo de combustible y los costos de operación y mantenimiento fueron obtenidos de la $\mathrm{EEH}$ y se utilizaron con el fin de ejecutar el análisis costobeneficio para esta investigación (ver Tabla n. ${ }^{\circ} 6$ ). Se realizó una comparación entre el "caso base" (tecnología tradicional usando luminarias HPS) y un "caso propuesto" (tecnología de energía limpia usando luminarias LED).

La tabla n. 7 muestra el resumen de los resultados de la hoja de cálculo obtenida en RETScreen utilizando el modelo de energía del caso propuesto. Se observa que el incremento del costo inicial por la sustitución de las lámparas tipo HPS con luminarias tipo LED es de USD 236,360. Sin embargo, el ahorro de costos de electricidad utilizando luminaria tipo LED es de USD 38,987 y además, se genera un ahorro por costos de operación y mantenimiento de USD 9,019. Según los resultados del estudio, el costo inicial por la adquisición de lámparas LED se recuperará al final del cuarto año. Por lo tanto, es posible concluir que, considerando el balance de costos y ahorros a lo largo de la vida de este proyecto, el proyecto es económicamente rentable y atractivo para inversores. En cuanto a la comparación del consumo de combustible y combustible ahorrado entre el caso base y el caso propuesto, se puede observar que mediante el uso de luminaria tipo LED se genera un ahorro de combustible del $53.3 \%$. 
Tabla n. ${ }^{\circ} 6$

Datos de entrada utilizados para el análisis costo-beneficio en RETScreen

\begin{tabular}{|l|c|}
\hline \multicolumn{1}{|c|}{ Datos de entrada } \\
\hline Tarifa eléctrica (USD/kWh) & 0.167 \\
\hline Horas en operación & 12 \\
\hline Dias en operación & 365 \\
\hline Tarifa escalada del costo de combustible (\%) & 4.74 \\
\hline Tasa de inflación (\%) & 2.45 \\
\hline Tasa de descuento (\%) & 6.3 \\
\hline Vida del proyecto (años) & 10 \\
\hline LED 200W (USD) & 1,000 \\
\hline LED 150W (USD) & 760 \\
\hline LED 70W (USD) & 300 \\
\hline Costo de operación y mantenimiento (USD/LED) & 29 \\
\hline
\end{tabular}

Fuente: Elaboración propia con datos del Banco Central de Honduras y la Empresa Energía de Honduras.

Tabla n. ${ }^{\circ} 7$

Resumen de resultados en RETScreen

\begin{tabular}{|c|c|c|c|c|c|c|c|}
\hline $\begin{array}{l}\text { Tipo de } \\
\text { Luminaria }\end{array}$ & $\begin{array}{l}\text { Electricidad } \\
\text { (MWh) }\end{array}$ & $\begin{array}{l}\text { Incremento } \\
\text { del costo } \\
\text { Inicial } \\
\text { (USD) }\end{array}$ & $\begin{array}{l}\text { Ahorro de } \\
\text { costos } \\
\text { (USD) }\end{array}$ & $\begin{array}{c}\text { Ahorro en } \\
\text { operación y } \\
\text { mantenimiento }\end{array}$ & $\begin{array}{l}\text { Año de } \\
\text { reembolso }\end{array}$ & $\begin{array}{l}\text { Costo } \\
\text { unidad de } \\
\text { luminaria } \\
\text { LED } \\
\text { (USD) }\end{array}$ & $\begin{array}{c}\text { Costo/LED } \\
\text { operación y } \\
\text { mantenimiento } \\
\text { (USD) }\end{array}$ \\
\hline 400W HPS & 233 & 236,360 & 38,537 & 9,019 & 4.92 & 760 & 29 \\
\hline Tipo & $\begin{array}{l}\text { Unidad de } \\
\text { consumo }\end{array}$ & $\begin{array}{l}\text { Consumo } \\
\text { (HPS) }\end{array}$ & $\begin{array}{l}\text { Costo } \\
\text { (HPS) } \\
\text { (USD) }\end{array}$ & $\begin{array}{l}\text { Consumo } \\
\text { (LED) }\end{array}$ & $\begin{array}{l}\text { Costo } \\
\text { (LED) } \\
\text { (USD) }\end{array}$ & $\begin{array}{l}\text { Ahorro } \\
\text { energético }\end{array}$ & $\begin{array}{l}\text { Ahorro } \\
\text { de costos } \\
\text { energéticos } \\
\text { (USD) } \\
\end{array}$ \\
\hline Electricidad & MWh & 437.8 & 73,109 & 204.3 & 34,123 & 233 & 38,987 \\
\hline \multicolumn{8}{|c|}{ ANALISIS DE COSTO } \\
\hline \multicolumn{3}{|l|}{ Tipo } & $\begin{array}{l}\text { Electricidad } \\
(\mathrm{MWh})\end{array}$ & Total (MWh) & \multicolumn{3}{|c|}{ Ahorro (\%) } \\
\hline Caso base & & HPS & 438 & 438 & \multirow{3}{*}{\multicolumn{3}{|c|}{$53.30 \%$}} \\
\hline Caso propues & & LED & 204 & 204 & & & \\
\hline \multicolumn{2}{|c|}{ Ahorro de combustible } & & 233 & 233 & & & \\
\hline \multicolumn{5}{|l|}{ Costo inicial } & \multicolumn{3}{|c|}{ USD 236,360} \\
\hline \multicolumn{8}{|l|}{ Costos anuales } \\
\hline \multicolumn{5}{|c|}{ Costos de operación y mantenimiento (ahorro) } & \multicolumn{3}{|l|}{ USD $-9,019$} \\
\hline \multicolumn{5}{|c|}{ Electricidad (caso propuesto) } & \multicolumn{3}{|c|}{ USD 34,123} \\
\hline \multicolumn{8}{|c|}{ Ahorros anuales } \\
\hline \multicolumn{5}{|c|}{ Electricidad (caso base) } & \multicolumn{3}{|c|}{ USD 73,109 } \\
\hline
\end{tabular}

Fuente: Elaboración propia utilizando los resultados de las hojas de cálculo de RETScreen. 
La tabla n. ${ }^{\circ}$, muestra las estimaciones de emisiones de gases de efecto invernadero del caso base y del caso propuesto. En el caso base, las emisiones estimadas ascienden a 145.3 $\mathrm{tCO}_{2}$ anualmente; por otro lado, en el caso propuesto las emisiones ascienden a $67.8 \mathrm{tCO}_{2}$ anualmente. Por lo tanto, el sistema de caso propuesto muestra una reducción de $77.5 \mathrm{tCO}_{2}$ emitidas al medio ambiente anualmente. En un periodo de 10 años las toneladas de dióxido

\section{Tabla.$^{\circ} 8$}

Análisis de emisiones de gases de efecto invernadero de carbono reducido se estiman en $775 \mathrm{tCO}_{2}$ utilizando luminarias tipo LED.

La Tabla n. 9 muestra el resumen financiero. Se puede observar que, para aproximadamente el quinto año, el proyecto comienza a ser rentable (ver Figura n. 9 ), debido a que se estima un reembolso simple a finales del cuarto año. Considerando que la empresa encargada de la gestión de distribución de la energía realizaría la inversión total para la compra de los sistemas de luminarias LED.

\begin{tabular}{|l|c|c|c|c|}
\hline \multicolumn{1}{|c|}{ Electricidad } & Mix & $\begin{array}{c}\text { Consumo de } \\
\text { electricidad }\end{array}$ & $\begin{array}{c}\text { Factor de emisión de gases de efecto } \\
\text { invernadero }\left(\mathrm{tCO}_{\mathbf{2}} / \mathbf{M W h}\right)\end{array}$ & $\begin{array}{c}\text { Emisión de gases de } \\
\text { efecto invernadero } \\
\left(\mathbf{t C O}_{2}\right)\end{array}$ \\
\hline Tipo & & & & 145.3 \\
\hline Sistema del caso base & $100 \%$ & 438 & 0.332 & 67.8 \\
\hline Sistema del caso propuesto & $100 \%$ & 204 & 0.332 & 77.5 \\
\hline \multicolumn{7}{|c|}{ Medida de eficiencia energética } \\
\hline \multicolumn{6}{|l}{ Reducción anual bruta de emisiones de gases de efecto invernadero $\left(\mathrm{tCO}_{2}\right)$} & \\
\hline
\end{tabular}

Fuente: Elaboración propia utilizando las hojas de cálculo de RETScreen.

Tabla n. ${ }^{\circ} 9$

Resumen financiero

\begin{tabular}{|l|c|}
\hline \multicolumn{2}{|c|}{ Ahorro y costo del proyecto } \\
\hline \multicolumn{1}{|c|}{ Costo inicial (USD) } & 236,360 \\
\hline \multicolumn{1}{|c|}{ Operación y mantenimiento (USD) } & \\
\hline \multicolumn{1}{|c|}{ Costo de combustible (caso propuesto) (USD) } & $-9,019$ \\
\hline \multicolumn{1}{|c|}{ Viabilidad financiera } & 34,123 \\
\hline Costo anual total (USD) & 25,104 \\
\hline Ahorro anual (USD) & 73,109 \\
\hline \multicolumn{2}{|c|}{} \\
\hline Equidad del TIR después de impuestos & $20.5 \%$ \\
\hline Activos del TIR después de impuestos & $20.5 \%$ \\
\hline Reembolso simple (años) & 4.9 \\
\hline Rentabilidad patrimonial (años) & 4.4 \\
\hline Valor presente neto (USD) & 196,689 \\
\hline Ahorros anuales del ciclo de duración (USD) & 27,105 \\
\hline Relación costo-beneficio (BCR) & 1.83 \\
\hline
\end{tabular}

Fuente: Elaboración propia utilizando las hojas de cálculo de RETScreen. 


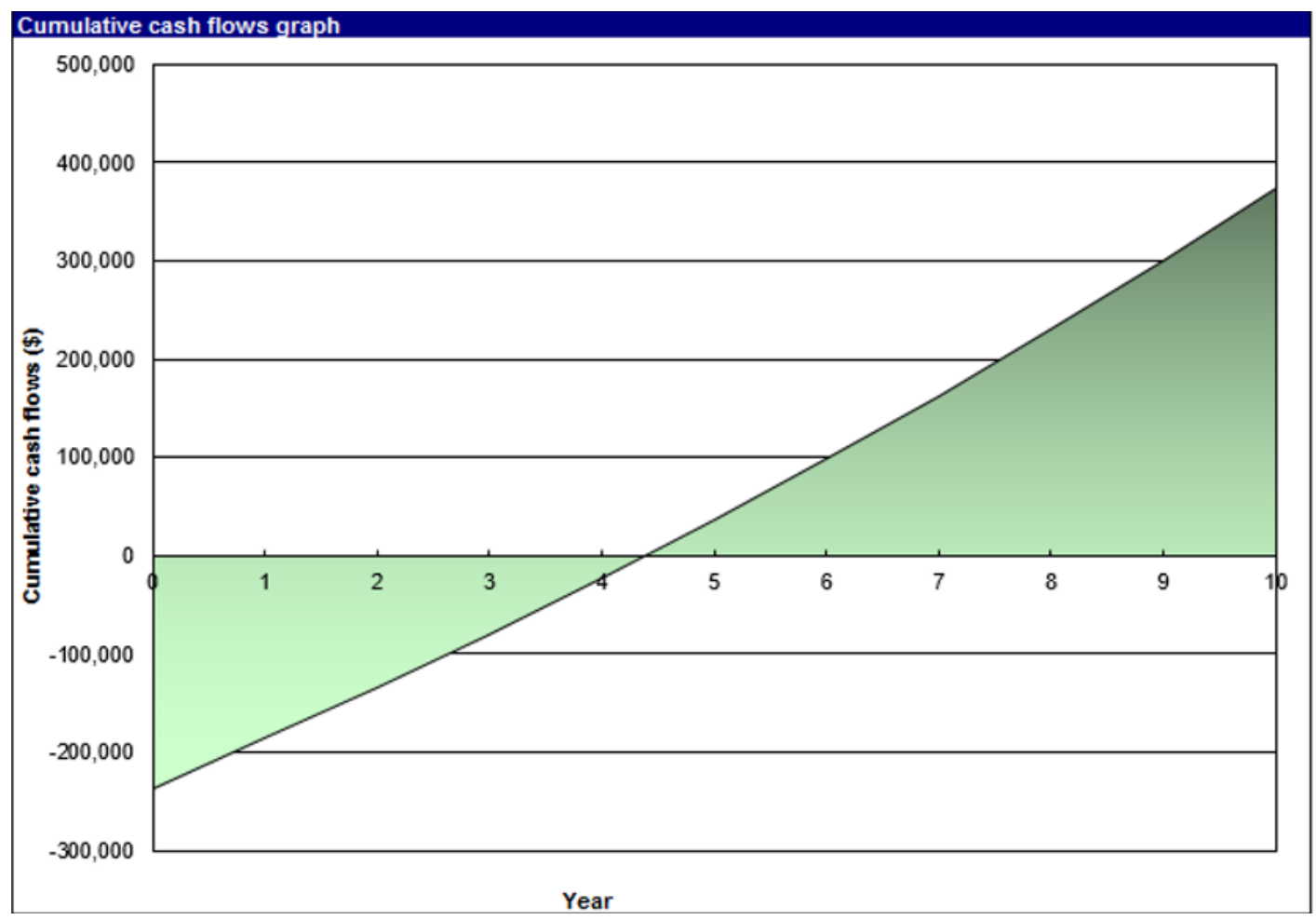

Figura $n .{ }^{\circ}$ 9. Flujos de caja acumulables. Fuente: Elaboración propia utilizando las hojas de cálculo de RETScreen.

E1 análisis financiero indica que la tasa interna de retorno (TIR) es del $20.5 \%$, siendo esta mayor que la tasa de descuento de $6.3 \%$; por lo tanto, el proyecto resulta atractivo para inversores. Además, el VPN estimado es de USD 196,689, la relación costo-beneficio (BCR) es de 1.83 , lo que implica que los costos del proyecto son compensados y se generaría un beneficio de USD 1.83 por cada dólar de los costos. Estos resultados, comprueban la viabilidad financiera del proyecto. Finalmente, se estimaron los ahorros anuales del ciclo de duración del caso propuesto, los cuales ascenderían a USD 27,105.

\section{Conclusiones}

Las luminarias tipo LED ofrecen una solución eficaz y cada vez más rentable para el desafío de la reducción del consumo de energía, reducción de la huella de carbono, reducción de gastos de la red de alumbrado público e incremento de la calidad de vida. Además, la utilización de luminarias LED demuestra tener algunos cobeneficios que le brindan valor agregado. Entre los cobeneficios a destacar se encuentran, reducción de la criminalidad como consecuencia de una mejor iluminación, mejora de la seguridad vial y reducción de accidentes, percepción pública positiva, desarrollo económico comunitario y 
revitalización urbana. Estos cobeneficios son importantes cuando se evalúan las luminarias LED no solo como una herramienta de ahorro de energía y reducción de la huella de carbono, sino como una herramienta para el desarrollo sostenible, ya que la iluminación vial repercute directamente en la calidad urbana.

Basado en el hecho de que las luminarias tipo HPS de 400W que se encuentran en la carretera CA-13 en La Ceiba, Honduras; se instalaron en 1994, ya han cumplido su vida útil y generan deficiente luminosidad; se concluye que es necesario cambiar el tipo de luminarias y se sugiere, basado en la viabilidad y rentabilidad del uso de luminarias LED, optar por ese sistema sin modificar las características de la carretera. Los resultados de la simulación llevada a cabo en el estudio muestran que el sistema LED de $150 \mathrm{~W}$ brinda los mayores niveles de iluminación, ahorro energético y reducción de emisiones de dióxido de carbono con relación al costo. La sugerencia de modificación requerirá 321 accesorios LED de $150 \mathrm{~W}$.

Aunque los costos iniciales de la retroadaptación de iluminación tradicional HPS a sistema de luminarias LED son altos, a través de la investigación, se demostró que los ahorros en gastos de energía y mantenimiento son significativos, siendo estos las principales motivaciones para la implementación de proyectos LED para el alumbrado público. Para el caso propuesto, anualmente se estima un ahorro energético de 233 MWh (53.3 \%) y ahorros que ascienden a USD 38,987.

Basado en el análisis económico realizado en esta investigación, se concluye que este es un proyecto viable. La recuperación de la inversión se alcanza al final del cuarto año, el VPN es de USD 196,689 con una TIR de $20.5 \%$ y un BCR de 1.83; los resultados muestran valores positivos, por lo tanto, el proyecto propuesto es financieramente rentable.

\section{Referencias}

Colon, C.J. (2010). Assessing the Economic and Environmental Impacts Associated with Currently Available Street Lighting Technologies. Air Force Institute of Technology, Ohio.

Comisión Internacional de la Iluminación. (2011). ILV: Vocabulario Internacional de Iluminación. Viena: Autor.

Dalton, C. (2012). What constitutes a good Tornado Diagram. Syncopation Software. Disponible en: https://www.syncopation.com/ blog/what-constitutes-good-tornado-diagram

Dewar, R.E., Olson, P.L. (2007). Human Factors in Traffic Safety. Estados Unidos: Lawyers \& Judges Publishing Company.

EU Smart Cities Information System. (2018). Smart street lighting. Smartcities-infosystem.eu

La Prensa de Honduras. (2018). Tarifa energética sufrirá aumento en septiembre. La Prensa de Honduras. Disponible en: https://www.laprensa. hn/economia/1209526-410/tarifa-energeticaenerg\%C3\%ADa-electrica-honduras-septiembre-

Maldonado, G.R. (2001). Instalaciones Eléctricas I. Cochabamba, Bolivia: Universidad Mayor de San Simón. 
Natural Resources Canada. (2018). RETScreen. Nrcan.gc.ca. Disponible en: https://www.nrcan. gc.ca/energy/software-tools/7417

Palisade. (1984). Monte Carlo Simulation. Palisade.com

Philips.(2017).Philips Lighting LED Professional Solutions. Lighting Philips. Disponible en: http:// www.lighting.philips.com/main/home

Tähkämö, L., Räsänen, R.-S., Halonen, L. (2016). Life cycle cost comparison of highpressure sodium and light-emitting diode luminaires in street lighting. Int. J. Life Cycle Assess. 21, 137-145. https://doi.org/10.1007/ s11367-015-1000-x
Wu, M.S., Huang, H.H., Huang, B.J., Tang, C.W., Cheng, C.W. (2009). Economic feasibility of solar-powered led roadway lighting. Renew. Energy. 34, 1934-1938. https://doi. org/10.1016/j.renene.2008.12.026

Zhou, H., Pirinccioglu, F., Hsu, P. (2009). A new roadway lighting measurement system. Transportation Research Part. C: Emerging Technologies. 17, 274-284. https://doi. org/10.1016/j.trc.2008.11.001 